\title{
Current transport in ramp-type junctions with engineered interface
}

\author{
J.-K. Heinsohn, R. Dittmann, ${ }^{\text {a) }}$ J. Rodríguez Contreras, J. Scherbel, A. Klushin, \\ and M. Siegel \\ Institut für Schicht- und Ionentechnik, Forschungszentrum Jülich GmbH, 52425 Jülich, Germany \\ C. L. Jia \\ Institut für Festkörperforschung, Forschungszentrum Jülich GmbH, 52425 Jülich, Germany \\ S. Golubov \\ Department of Applied Physics, University of Twente, Enschede, The Netherlands \\ M. Yu. Kupryanov \\ Institute of Nuclear Physics, Moscow State University, Moscow, Russia
}

(Received 27 September 2000; accepted for publication 21 December 2000)

\begin{abstract}
The transport properties of "interface-engineered" edge-type $\mathrm{YBa}_{2} \mathrm{Cu}_{3} \mathrm{O}_{7}$ Josephson junctions are investigated in detail. We have investigated the dependence of the current-voltage characteristics on external magnetic field, temperature, and microwave irradiation and compare them to the resistively shunted junction model. The temperature dependence of the critical current and the normal resistance allows us to draw conclusions to the transport of quasiparticles and Cooper pairs in the investigated "interface-engineered" junctions. We have studied the properties of junctions for which $\mathrm{La}$ doped $\mathrm{YBa}_{2} \mathrm{Cu}_{3} \mathrm{O}_{7}$ is used for the superconducting electrodes. We will propose a model for the undoped and the La doped case which takes into account a barrier which consists of a series connection of a normal conducting layer and an insulator, containing superconducting microconstrictions. (C) 2001 American Institute of Physics. [DOI: 10.1063/1.1351056]
\end{abstract}

\section{INTRODUCTION}

Interface-engineered junctions (IEJs) are an interesting approach for the realization of reliable, controllable, hightemperature superconducting (HTS) Josephson junctions. The idea of fabricating a barrier by interface treatments instead of using an epitaxially grown nonsuperconducting thin film was first suggested by Moeckly et al. ${ }^{1}$ At present, several groups are employing IEJs by using different approaches for the interface treatment. ${ }^{2-7}$ All approaches have in common an ion treatment of the surface of the base electrode in the ramp area and a subsequent annealing step, but the methods for the generation of the ions and the process parameters differ strongly from group to group. However, each group claims that the junctions are much more reliable than ramptype junctions with epitaxially deposited barrier layers, previously fabricated in their laboratories.

Since IEJs are resistively shunted junctions the most suitable model to describe their transport properties is the resistively and capacitively shunted junction (RCSJ) model. ${ }^{8}$ For most cryoelectronic applications, e.g., superconducting quantum interference devices, digital circuits, or voltage calibrators, RSCJ-like junctions are required. Nevertheless, no detailed comparisons of the static and dynamic properties of IEJs to the RCSJ model at different temperatures have been published up to now.

The current transport and the nature of the barrier are still open questions since the results of microstructural investigations differ from group to group. Whereas some groups

a) Author to whom correspondence should be addressed; phone: +49-2461612357, fax: +49-2461-612940, electronic mail: r.dittmann@fz-juelich.de observe the formation of a cation disordered (pseudo) cubic $\mathrm{YBa}_{2} \mathrm{Cu}_{3} \mathrm{O}_{7}(\mathrm{YBCO})$ phase, ${ }^{9,10}$ Wen et al. ${ }^{11}$ report on the formation of a $\mathrm{Cu}$ deficient $\mathrm{YBCO}$ phase $\mathrm{Y}_{1-x} \mathrm{BaCu}_{x} \mathrm{O}_{y}$. There exists no consistent picture for the current transport. The published current densities, the normal resistances, and the suggested transport mechanisms differ for the junctions fabricated in different ways. Some groups observe properties of an insulating barrier containing localized states ${ }^{4,5}$ whereas previous results of our group hint on a metallic behavior of the barrier material. ${ }^{6}$

The presence of La either in the superconducting electrodes or in the insulator strongly influences the properties of the junctions. Hunt et al. observed a strong increase of the normal resistance and a decrease of the critical current by using La doped YBCO for the bottom electrode. ${ }^{2}$ Satoh et al. observed RCSJ-like current-voltage $(I-V)$ characteristics only if $\mathrm{La}$ is present in the near area of the ramp. ${ }^{5}$

We will present detailed measurements of the transport properties of IEJs with $\mathrm{YBa}_{2} \mathrm{Cu}_{3} \mathrm{O}_{7}$ (undoped junctions) as well as $\mathrm{YBa}_{1.95} \mathrm{La}_{0.05} \mathrm{Cu}_{3} \mathrm{O}_{7}$ electrodes $[\mathrm{La}(5 \%)$ doped junctions]. The $I-V$ characteristics as well as the dependence of the critical current $I_{C}$ on external magnetic field, $I_{C}(H)$, were investigated for the whole temperature range below the superconducting transition temperature $T_{C}$. The $I-V$ characteristics and their response to microwave irradiation will be compared with the RCSJ model and the deviations will be discussed. We will propose a model to explain the transport of quasiparticles and Cooper pairs in undoped and $\mathrm{La}(5 \%)$ doped junctions and draw conclusions to the nature of the barrier. 


\section{JUNCTION PREPARATION}

The junction preparation process can be divided into the deposition of a $\mathrm{YBCO}-\mathrm{SrTiO}_{3}$ bilayer, the ramp fabrication process, the interface engineering, and the deposition of the counterelectrode.

First, a bilayer consisting of a $120 \mathrm{~nm}$ thick YBCO or $\mathrm{YBa}_{1.95} \mathrm{La}_{0.05} \mathrm{Cu}_{3} \mathrm{O}_{7}[\mathrm{La}(5 \%)$ doped $\mathrm{YBCO}]$ film and a 220 $\mathrm{nm}$ thick $\mathrm{SrTiO}_{3}$ (STO) film is deposited in situ by pulsed laser deposition. We employed STO as well as $\mathrm{LaAlO}_{3}$ as substrate materials and did not observe any influence on the film quality or junction properties. The YBCO films are deposited at a substrate temperature of $T=805^{\circ} \mathrm{C}$, an oxygen pressure $p=60 \mathrm{~Pa}$, an energy density of $2 \mathrm{~J} / \mathrm{cm}^{2}$, and a laser frequency of $10 \mathrm{~Hz}$. The STO films deposited in situ on top of the YBCO films are fabricated at $T=760{ }^{\circ} \mathrm{C}, p=15 \mathrm{~Pa}$, with the same energy density and frequency as for YBCO. Further details can be extracted from Ref. 12.

For the fabrication of the ramps, the bilayers are patterned by conventional photolithography. The STO film is Ar-ion-beam milled in a Kaufmann-type source using a current density of $0.25 \mathrm{~mA} / \mathrm{cm}^{2}$ and an energy of $250 \mathrm{eV}$. The sample is tilted $30^{\circ}$ to the substrate normal and rotates during the etching process. The sample is cooled down to $-15^{\circ} \mathrm{C}$ during ion beam etching. The photoresist is then removed in an oxygen plasma. Subsequently, using the STO ramp as an etching mask, the YBCO ramps are ion-beam milled at the same etching parameters. This process results in ramps with edge angles of $20^{\circ}$ for STO and $30^{\circ}$ for YBCO. The thickness of the STO layer is reduced to $20-50 \mathrm{~nm}$ during the ion milling of the YBCO ramp. A more detailed description of the ramp fabrication process can be found in Refs. 12 and 13.

The interface engineering consists of a short ex situ ionmilling step at higher voltages and an annealing step in the deposition chamber. We investigated the parameters for these steps in combination with the deposition temperature of the counterelectrode using statistical methods. ${ }^{12}$ In summary, we found out that the homogeneity of the junctions can be improved by using higher etching voltages and lower annealing temperatures whereas the critical currents of the junctions are mainly determined by the deposition temperature of the counterelectrode. Optimal junction properties are achieved by using a $5 \mathrm{~min}$ annealing step at $1200 \mathrm{~V}$, a 30 min annealing step at $500^{\circ} \mathrm{C}$ at the deposition pressure of $60 \mathrm{PaO}_{2}$, and a deposition temperature of $760^{\circ} \mathrm{C}$ for the counterelectrode.

The junctions with bridge widths between 1 and $8 \mu \mathrm{m}$ are patterned by conventional photolithography and Ar-ionbeam milling. Finally, a $200 \mathrm{~nm}$ thick gold layer is evaporated and patterned by a lift-off process to provide electrical contacts.

Several undoped and $\mathrm{La}(5 \%)$ doped junctions were investigated by transmission electron microscopy (TEM) and no significant difference between the microstructure of the junctions was observed. The TEM micrograph of a $\mathrm{La}(5 \%)$ doped junction is shown in Fig. 1. At the interface between the bottom YBCO electrode and the counterelectrode a region with a slightly different contrast can be identified. However, in higher resolution, as shown in the inset of Fig. 1, no

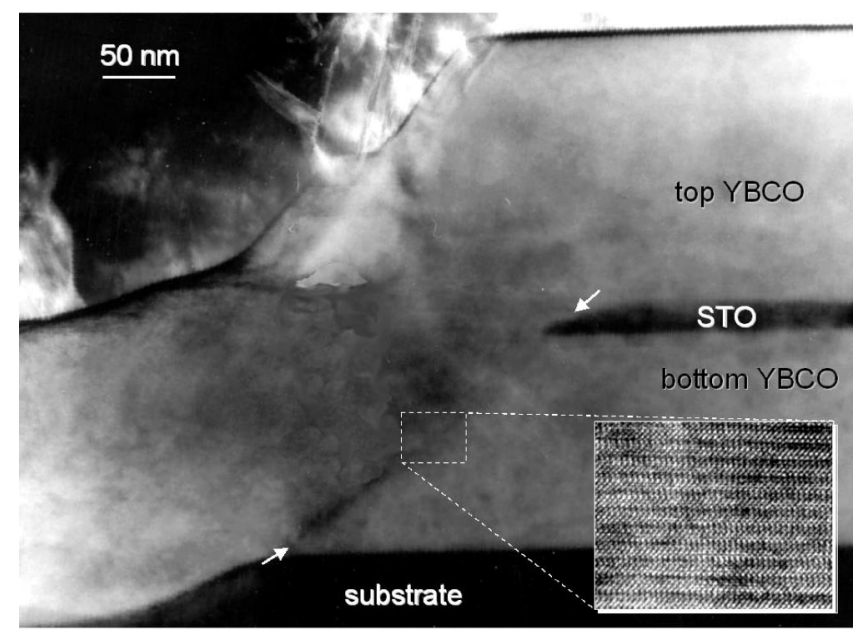

FIG. 1. TEM micrograph of the junction area. The arrows indicate the interface between the top and bottom electrode, which can be identified by a region with different contrast. The inset shows a high resolution image of the interface region. The thickness of the STO film is reduced to about 20 $\mathrm{nm}$ during the ion milling of the YBCO ramp.

interface layer with a different crystalline structure can be observed. This is in contradiction to the observations published in Refs. 9 and 11, where a 2 to $3 \mathrm{~nm}$ thin interface layer with a clearly different crystal structure was detected.

Cation disorder in the interface region due to the Ar-ion treatment could lead to local strain which may become visible in TEM as a region with different contrast. So the TEM investigations of our junctions are consistent with an interface layer where the orthorhombic crystal structure of YBCO is maintained, but in which some cation disorder is present.

\section{STATIONARY PROPERTIES}

The chip layout used for our investigations contains 40 junctions with 2-4 $\mu \mathrm{m}$ junction width. We investigated about ten chips with undoped junctions and about ten chips with La doped junctions with different La content. Since our process ensures the reliable fabrication of Josephson junctions, the measurements presented in this article are representative of our IEJs. The Josephson junctions are measured in the standard four-point geometry. A coil situated in our probe enables us to measure the $I-V$ characteristics in magnetic fields up to $3 \mathrm{mT}$. Trapping of flux is a crucial point in ramp-type junctions. Marx et al. showed by lowtemperature-scanning-electron microscopy that magnetic flux is trapped in the YBCO film covering the ramp edge. ${ }^{14}$ Therefore the junctions are shielded against external magnetic fields by Cryoperm shielding. Since self-generated fields also induce trapped magnetic flux in the junctions, the maximum critical current is often obtained at nonzero magnetic fields. Therefore we adjusted the magnetic field towards the maximum of the critical current before we recorded the $I-V$ characteristics. A detailed investigation of the magnetic-field dependence of the $I-V$ characteristics of our junctions will be published elsewhere.

The normal resistance $R_{N}$ is determined by the slope of the linear part of the $I-V$ characteristics at high bias currents where $V \gg I_{C} R_{N}$. 

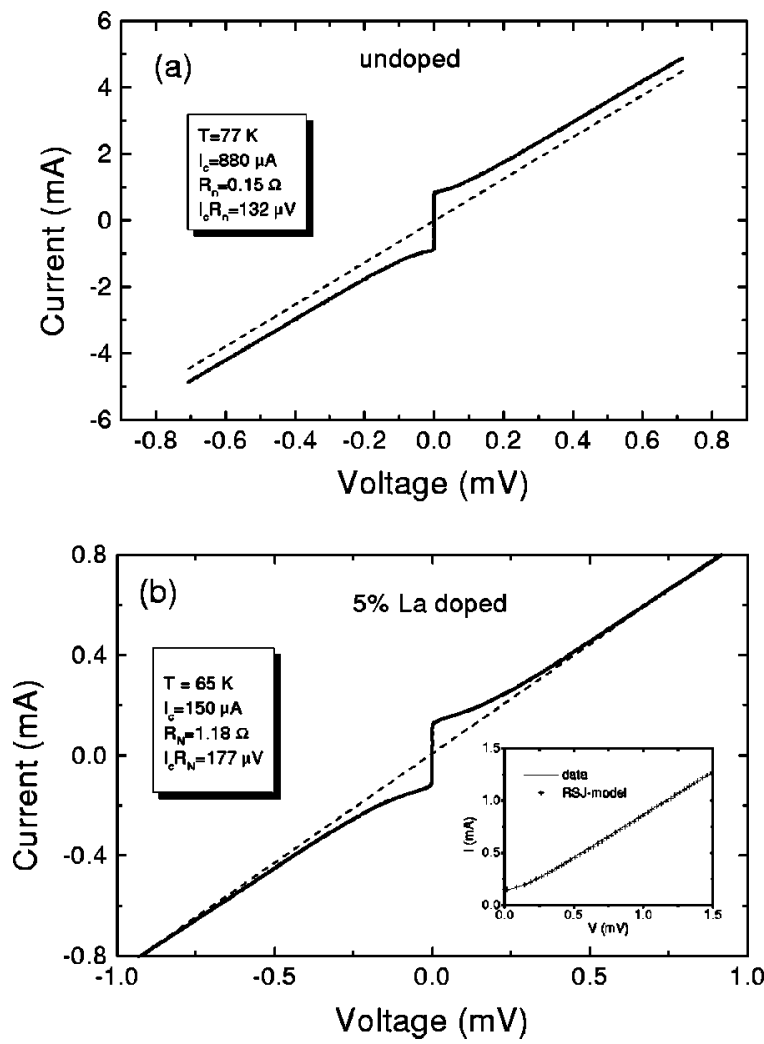

FIG. 2. (a) $I-V$ characteristic of a $4 \mu \mathrm{m}$ wide undoped junction at $77 \mathrm{~K}$. (b) $I-V$ characteristic of a $3 \mu \mathrm{m}$ wide $\mathrm{La}(5 \%)$ doped junction at $65 \mathrm{~K}$.

\section{A. Influence of La doping on the junction properties}

Figure 2(a) shows a typical $I-V$ characteristic of an undoped junction at $77 \mathrm{~K}$. The parameters can be extracted from Table I. The shape of the $I-V$ characteristic is RCSJlike but approximates at high bias current a straight line with a positive offset relative to the $I-V$ characteristic of an ohmic resistor [see the dashed line in Fig. 2(a)]. This offset corresponds to an excess current of about $500 \mu \mathrm{A}$.

For $\mathrm{La}(5 \%)$ doped junctions, supercurrents become detectable between 75 and $80 \mathrm{~K}$ although the critical temperatures of the $\mathrm{La}(5 \%)$ doped YBCO films are only slightly suppressed (at most 1 to $2 \mathrm{~K}$ ). Figure 2(b) shows a typical $I-V$ characteristic of a $\mathrm{La}(5 \%)$ doped junction at $65 \mathrm{~K}$. The shape can be well-described by the RCSJ model with neglected capacitance as can be seen in the inset of the figure.

The parameters of the undoped and the $\mathrm{La}(5 \%)$ doped junctions presented in Fig. 2 are summarized in Table I. It shows that the critical current density $j_{C}$ of the $\mathrm{La}(5 \%)$ doped junctions is about one order of magnitude lower and

TABLE I. Summary of the junction parameters of the junctions in Fig. 2 ( $j_{C}$ : critical current density; $R_{N} A$ : normal resistance times junction area; and $T$ : measurement temperature).

\begin{tabular}{cccccc}
\hline \hline $\begin{array}{c}\text { Sample } \\
\text { No. }\end{array}$ & $\begin{array}{c}\text { La } \\
\text { concentration }\end{array}$ & $T(\mathrm{~K})$ & $j_{C}\left(\mathrm{~A} \mathrm{~cm}^{-2}\right)$ & $R_{N} A\left(\Omega \mathrm{cm}^{2}\right)$ & $I_{C} R_{N}(\mu \mathrm{V})$ \\
\hline 1 & $0 \%$ & 77 & $1.8 \times 10^{5}$ & $7.2 \times 10^{-10}$ & 132 \\
1 & $0 \%$ & 65 & $2.5 \times 10^{5}$ & $6.2 \times 10^{-10}$ & 155 \\
2 & $5 \%$ & 65 & $4.2 \times 10^{4}$ & $4.2 \times 10^{-9}$ & 177 \\
\hline \hline
\end{tabular}

the $R_{N} A$ is about one order of magnitude higher than that for undoped junctions. Similar results have been obtained by other groups. $^{2}$

Since a high concentration of $\mathrm{La}$ reduces the $T_{C}$ of YBCO, ${ }^{15}$ a possible explanation for the difference between undoped and $\mathrm{La}(5 \%)$ doped junctions could be that La diffuses into the ramp area and this agglomeration of La results in an interface layer with reduced $T_{C}$ forming an additional barrier.

From this point of view, it seems possible to adjust the junction parameters by the La content. Therefore we investigated junctions fabricated with electrodes of doping levels from $0 \%$ to $7 \%$. We identified two groups of junctions: For $\mathrm{La}(5 \%)$ and $\mathrm{La}(7 \%)$ doped junctions, the critical currents are about one order of magnitude lower and the normal resistances are about one order of magnitude higher than for the $\mathrm{La}(3 \%)$ doped and undoped junctions. This means that a threshold La content is needed to change the junction parameters, but it is impossible to continuously adjust the junction parameters. Due to these results, it seems unlikely that the La content itself determines the electrical properties of the barrier. However, $\mathrm{La}$ is prerequisite for the modification of the barrier layer.

To further investigate the role of $\mathrm{La}$ in the preparation process, we fabricated junctions where $\mathrm{La}(5 \%)$ doped $\mathrm{YBCO}$ was used either only for the base electrode or only for the counterelectrode. The junctions with $\mathrm{La}(5 \%)$ doped $\mathrm{YBCO}$ bottom electrodes behave like junctions in which both electrodes consist of $\mathrm{La}(5 \%)$ doped $\mathrm{YBCO}$. The junctions with $\mathrm{La}(5 \%)$ doped YBCO top electrodes behave like undoped junctions. This shows that the interdiffusion of $\mathrm{La}$ is not the most important mechanism for the barrier formation in the $\mathrm{La}(5 \%)$ doped junctions because interdiffusion would as well occur during the fabrication of the counterelectrode. We conclude that La has to be present during etching and annealing to influence the formation of the barrier.

Hunt et $_{\text {al. }}{ }^{2}$ suggest that during the treatment of the ramp, the La atoms interchange in the interface region with a much higher probability with $\mathrm{Y}$ than $\mathrm{Ba}$ atoms, because the atomic radius of $\mathrm{La}$ is more similar to $\mathrm{Y}$ as the atomic radius of Ba. Wen et al. observed by TEM that if they utilize the La containing insulator $\left(\mathrm{La}_{0.3} \mathrm{Sr}_{0.7}\right)\left(\mathrm{Al}_{0.65} \mathrm{Ta}_{0.35}\right) \mathrm{O}_{3}$ the barrier is continuous whereas for $\mathrm{SrTiO}_{3}$ a high number density of pinholes is observed. ${ }^{11}$

We did not observe a difference between undoped and $\mathrm{La}(5 \%)$ doped junctions by TEM. The nature of the barrier for the different types of junctions will be discussed in the context of the transport properties of our junctions in Sec. V.

\section{B. Behavior of the junctions in different current density regions}

Due to the increase of the critical current with decreasing temperature the short-junction limit where self-field effects of the critical current can be neglected may be exceeded at lower temperatures. Therefore we measured $I-V$ characteristics and $I_{C}(H)$ patterns for several samples for different temperatures below $T_{C}$. To check the role of self-field effects due to Joseph-son currents in our junctions, we regard the relation of the junction width $w$ to the Josephson penetra- 

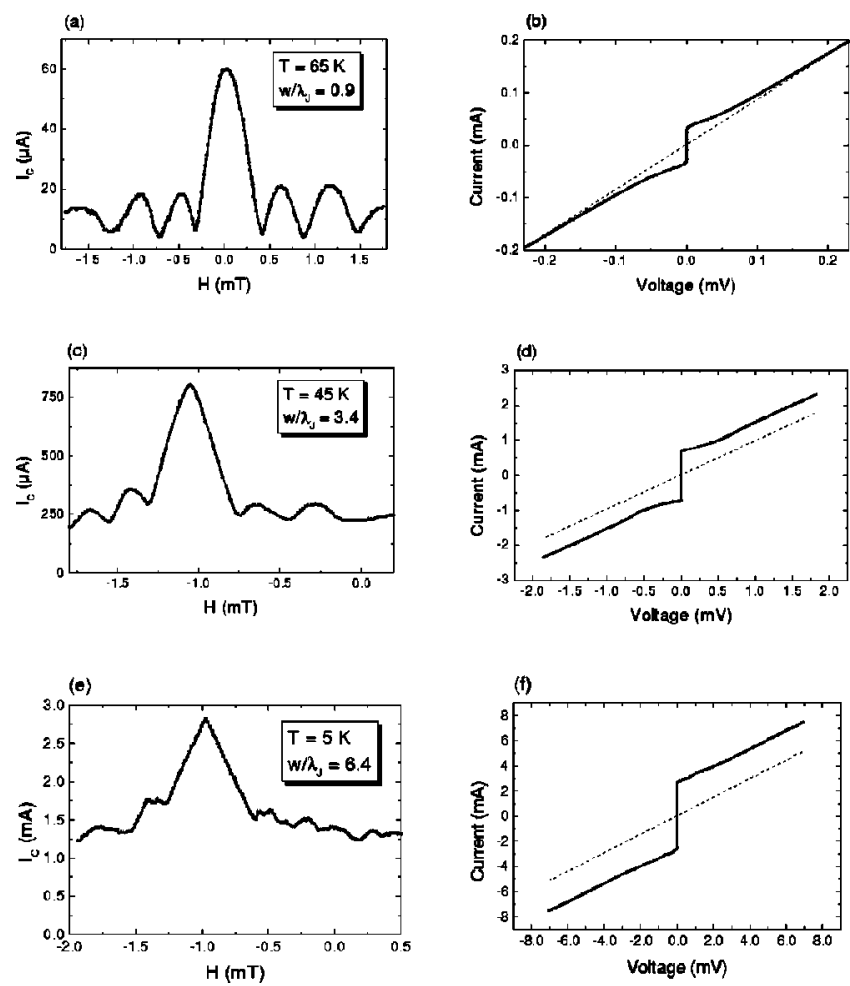

FIG. 3. $I_{C}(H)$ patterns and $I-V$ characteristics of a $3 \mu \mathrm{m}$ wide $\mathrm{La}(5 \%)$ doped junction at different temperatures: (a) and (b): $65 \mathrm{~K}$; (c) and (d): 45 $\mathrm{K}$; (e) and (f): $5 \mathrm{~K}$. The dashed lines correspond to the Ohmic law with the respective $R_{N}$ of the junction.

tion depth $\lambda_{J}=\sqrt{\hbar / 2 e \mu_{0} d^{\prime} j_{C}}$, with $d^{\prime}=d+2 \lambda_{L}$ (see, e.g., Ref. 16). We used $d^{\prime}=2 \lambda_{L, a b}=300 \mathrm{~nm}$ because supercurrents flow in the $a b$ planes and the thickness of our barrier layer can be neglected $\left(\lambda_{L} / d \gg 1\right.$ in any case).

Figure 3 shows the $I_{C}(H)$ patterns with corresponding $I-V$ characteristics of a $3 \mu \mathrm{m} \mathrm{La}(5 \%)$ doped junction at different current density regions. At $65 \mathrm{~K}$, the critical current density is $j_{C}=8.5 \times 10^{3} \mathrm{~A} / \mathrm{cm}^{2}$ corresponding to $w / \lambda_{J}=0.9$. Therefore the junction is clearly in the short-junction limit. The $I_{C}(H)$ curve is very similar to the Fraunhofer pattern [see Fig. 3(a)]. Deviations from the ideal Fraunhofer pattern may be attributed to barrier inhomogeneities. The $I-V$ characteristic can be well-described by the RCSJ model and no significant amount of excess current is observed [see Fig. 3(b)].

At $45 \mathrm{~K}$, the critical current density is $j_{C}=1.1$ $\times 10^{5} \mathrm{~A} / \mathrm{cm}^{2}$ corresponding to $w / \lambda_{J}=3.4$ which means that the junction is in the transition to the long-junction regime. There appears an excess current in the $I-V$ characteristics [see Fig. 3(d)] and the influence of self-field effects can be seen in the $I_{C}(H)$ curves: the second maxima are suppressed and the central maximum approaches a triangular shape [see Fig. 3(c)].

At $5 \mathrm{~K}$, the current density is $j_{C}=3.9 \times 10^{5} \mathrm{~A} / \mathrm{cm}^{2}$ corresponding to $w / \lambda_{J}=6.4$. The long-junction characteristics are obvious in Figs. 3(e) and 3(f). The $I_{C}(H)$ characteristic is triangular, the second maxima are nearly completely suppressed as expected for a long junction. ${ }^{16}$ Furthermore, there is almost $100 \%$ excess current in the $I-V$ characteristic [Fig. 3(f)]. The RCSJ model is not valid anymore.

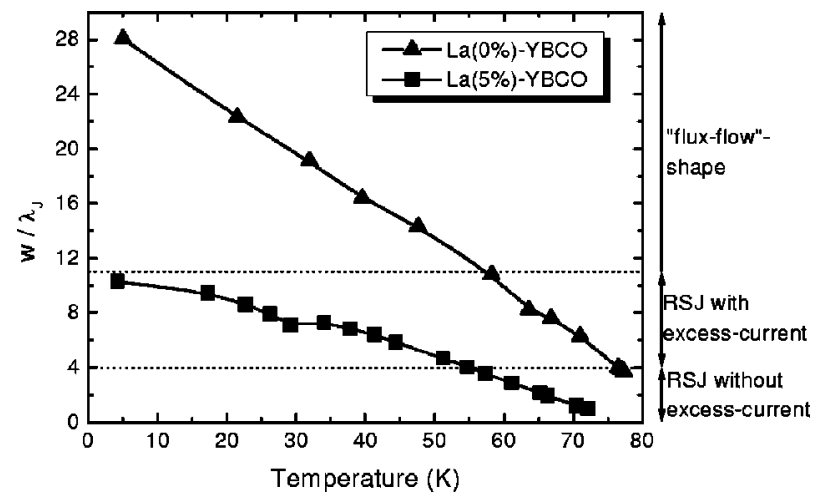

FIG. 4. Temperature dependences of $w / \lambda_{J}$ for a $4 \mu \mathrm{m}$ wide undoped junction and a $3 \mu \mathrm{m}$ wide $\mathrm{La}(5 \%)$ doped junction. The dotted horizontal lines indicate the $w / \lambda_{J}$ ranges where different shapes of the $I-V$ characteristics are observed in the junctions.

Since barrier inhomogeneities may cause additional deviations from a homogeneous current distribution, there is no strict value of $w / \lambda_{J}$ for which junctions switch from short junction to long junction behavior. Furthermore, although the ramp geometry is very similar to an overlap junction configuration, the electrodes are in the same plane and the boundary conditions may be different. Therefore a quantitative comparison of our $I_{C}(H)$ curves to existing calculations is not reasonable.

Nethertheless, some features of the junctions demonstrated in Fig. 3 are typical for all our junctions in the different $j_{C}$ regions. At $w / \lambda_{J} \approx 4$, excess current becomes significant in the $I-V$ characteristics. Since undoped junctions and $\mathrm{La}(5 \%)$ doped junctions have different current densities, $w / \lambda_{J}=4$ is obtained at different temperatures. To illustrate this effect, Fig. 4 shows the temperature dependence of $w / \lambda_{J}$ for an undoped junction and a $\mathrm{La}(5 \%)$ doped junction. The temperature range can be divided into three regions of $w / \lambda_{J}$. For $w / \lambda_{J}>4$, excess current becomes significant in the $I-V$ characteristics. For $w / \lambda_{J}>11$, the $I-V$ characteristics have a positive curvature like flux-flow $I-V$ characteristics. In the case of $\mathrm{La}(5 \%)$ doped junctions, the junctions are RCSJ-like in the whole temperature range, but start to have a significant amount of excess current at $50 \mathrm{~K}$. In the case of undoped junctions, the $I-V$ characteristics show ideal RCSJ behavior only in the vicinity of $T_{C}$. Below $55 \mathrm{~K}$, the $I-V$ characteristics are already flux-flow-like. Therefore undoped junctions are not suitable for applications where ideal RCSJ behavior is needed at temperatures below $77 \mathrm{~K}$.

Waldram et al. showed that for $w / \lambda_{J}>4$ a dc supercurrent persists up to large voltages. ${ }^{17}$ This supercurrent can be identified with our excess current $I_{\mathrm{ex}}$ because the voltage regime where the supercurrent should disappear is not obtained in the $I-V$ characteristics before dissipation in the superconducting electrodes leads to a positive curvature. The amount of additional supercurrent is expected to increase with increasing $w / \lambda_{J}$ as experimentally observed in lowtemperature-superconductor junctions. ${ }^{17}$ Therefore it is reasonable to attribute the increase of the excess current in our junctions with $w / \lambda_{J}$ to self-field effects. This is in agreement 

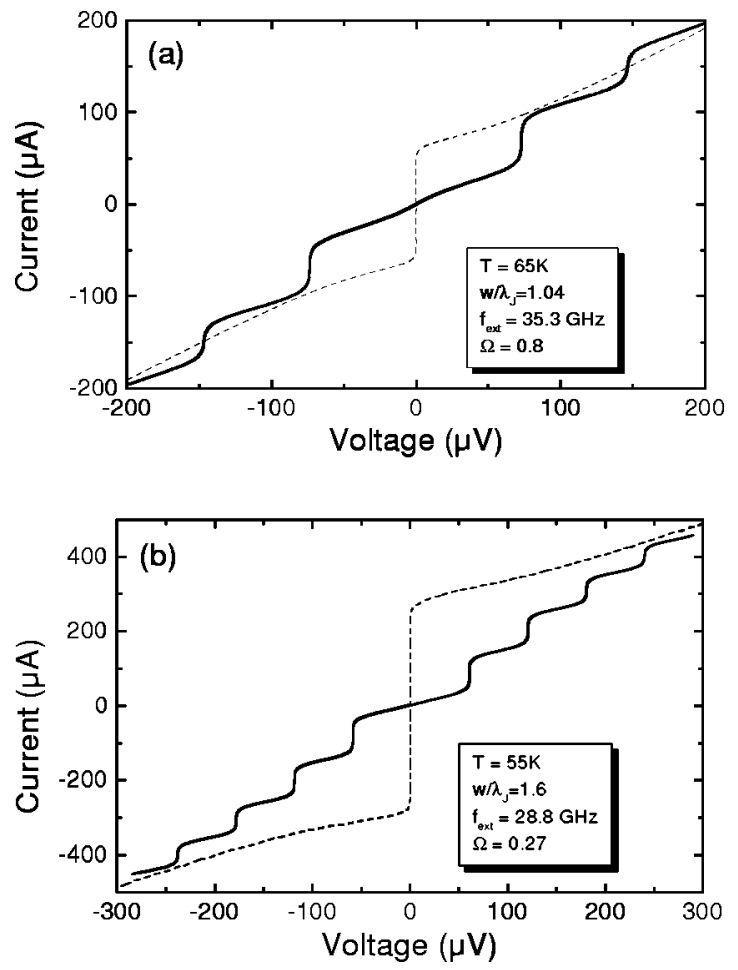

FIG. 5. $I-V$ characteristics of a $3 \mu \mathrm{m}$ wide $\mathrm{La}(5 \%)$ doped junction (a) at 65 $\mathrm{K}$ and $(\mathrm{b})$ at $55 \mathrm{~K}$. The dashed lines are the $I-V$ characteristics without microwave irradiation. The continuous lines correspond to the $I-V$ characteristics at (a) $35.3 \mathrm{GHz}$ and (b) $28.8 \mathrm{GHz}$.

with the increase of $I_{\mathrm{ex}} / I_{C}$ with decreasing temperature which is observed in our junctions.

Concerning the observation of flux-flow-like $I-V$ characteristics at high current densities $\left(w / \lambda_{J}>11\right)$, heating effects as well as dissipation in the electrodes may be an explanation, especially at high temperatures. Furthermore, the $T_{C}$ of a possible interlayer with reduced critical temperature may be exceeded in the corresponding temperature regime. However, it is not unreasonable to explain the observation of flux-flow-like $I-V$ characteristics with the dissipation of Josephson vortices in the junctions.

\section{DYNAMIC PROPERTIES}

To compare the dynamic properties of our junctions with the RCSJ model at different characteristic frequencies $2 e I_{C} R_{N} / h$, we investigated the $I-V$ characteristics of our junctions under microwave irradiation at two different temperatures. Since the $\mathrm{La}(5 \%)$ doped junctions are in the shortjunction limit for a broader temperature range than the undoped junctions, we measured the microwave response of a $\mathrm{La}(5 \%)$ doped junction at 65 and $55 \mathrm{~K}$.

Figure 5(a) shows the $I-V$ characteristics of a $3 \mu \mathrm{m}$ wide junction with and without microwave irradiation at 65 $\mathrm{K}$. With $w / \lambda_{J}=1.04$, the junction is clearly in the shortjunction regime and no significant excess current is visible. The $I-V$ characteristic exhibits well-defined Shapiro steps under microwave irradiation with a frequency $f_{\mathrm{ext}}$. The characteristic voltage $I_{C} R_{N}$ is $92 \mu \mathrm{V}$ which results at $f_{\text {ext }}$ $=35.5 \mathrm{GHz}$ in a normalized frequency of $\Omega=h f_{\text {ext }} / 2 e I_{C} R_{N}$ $=0.8$.
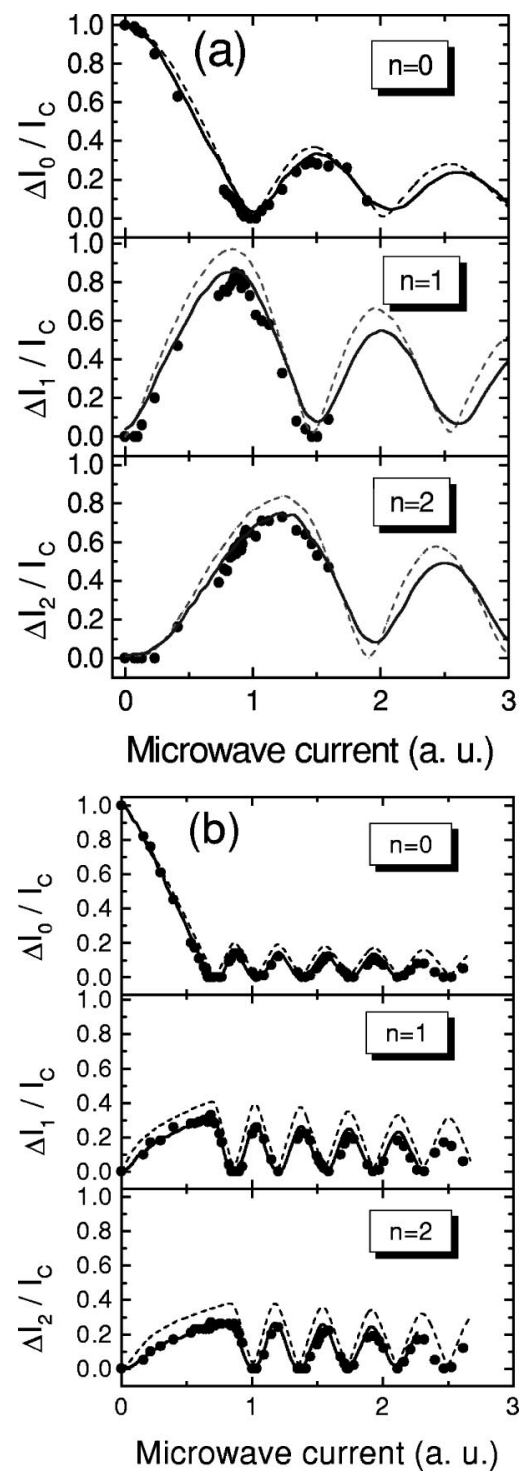

FIG. 6. Dependence of the Shapiro-step heights on the microwave current for $n=0,1,2$ of the junction shown in Fig. 4. (a) $T=65 \mathrm{~K}$ and $f_{\text {ext }}$ $=35.3 \mathrm{GHz}$. The dashed line shows the simulation without noise for $\Omega$ $=0.79$ and $\beta_{C}=0.1$; the continuous line shows the simulation with noise parameter $\Gamma=0.036$. (b) $T=55 \mathrm{~K}$ and $f_{\text {ext }}=28.8 \mathrm{GHz}$. The dashed line shows the simulation without noise for $\Omega=0.23$ and $\beta_{C}=0.1$; the continuous line shows the simulation with noise parameter $\Gamma=0.016$.

Figure 5(b) shows the $I-V$ characteristic of the junction with and without Shapiro steps at $55 \mathrm{~K}$. With $w / \lambda_{J}=1.6$, the junction is still in the short-junction regime, but some contribution of excess current to the critical current exists. The characteristic voltage $I_{C} R_{N}$ is $270 \mu \mathrm{V}$ which results at $f_{\text {ext }}$ $=28.8 \mathrm{GHz}$ in $\Omega=0.27$.

Figure 6 shows the dependence of the heights of the Shapiro steps on the microwave current at 65 and $55 \mathrm{~K}$. Additionally, in Fig. 6(a) the results of simulations with the RCSJ model with $\Omega=0.79$ and $\beta_{C}=2 e I_{C} R_{N}^{2} C / \hbar=0.1(C$ is the capacitance of the junction) without noise (dashed line) and with the influence of noise (straight line) are plotted. With a noise parameter $\Gamma=0.036$, the data at $65 \mathrm{~K}$ can be well-described with the RCSJ model. At $65 \mathrm{~K}$, the values for $\Omega$ and $\Gamma$ correspond exactly to the values calculated from the 
measured $I_{C} R_{N}$ product and from thermal fluctuations, $\Gamma$ $=2 e k_{B} T / \hbar I_{C}$, respectively.

In Fig. 6(b) the results of simulations with the RCSJ model with $\Omega=0.23$ and $\beta_{c}=0.1$ without noise (dashed line) and with the influence of thermal noise (straight line) are plotted. With a noise parameter $\Gamma=0.016$, the data can be well-described with the RCSJ model. This value is higher than the noise parameter calculated from thermal fluctuations, $\Gamma=0.009$, at $55 \mathrm{~K}$. There is also a discrepancy between the $\Omega=0.27$ determined from the static $I-V$ characteristics and the $\Omega=0.23$ used for the simulation of the Shapiro steps at $55 \mathrm{~K}$. Therefore at lower temperatures, the values for $I_{C}$ and $R_{N}$ determined from the static $I-V$ characteristics are not exactly suitable for the description of the dynamics of the junctions. A possible explanation is that with decreasing temperature additional transport channels influence $I_{C}$ and $R_{N}$ but do not contribute to the dynamics of the junctions.

For both investigated internal frequencies, corresponding to temperatures where the junction is in the short-junction regime, the dynamic properties qualitatively can be described with the RCSJ model. For lower temperatures, for which $w / \lambda_{J}>4$, we observe subharmonic steps in the $I-V$ characteristics of this junction as predicted for Josephson junctions in the long-junction regime. ${ }^{17}$

\section{CURRENT TRANSPORT PROPERTIES}

The temperature dependencies of $R_{N}$ and $I_{C}$ allow one to draw conclusions to the transport of quasiparticles and Cooper pairs. Therefore we measured $R_{N}(T)$ and $I_{C}(T)$ for several undoped and $\mathrm{La}(5 \%)$ doped samples. In Sec. V A we present typical measurements of each junction type. In Sec. VB we propose a model which consistently explains the experimental results.

\section{A. Temperature dependence of $I_{C}$ and $R_{N}$}

An important means to draw conclusions to the transport of quasiparticles is to investigate the temperature dependence of the normal resistance of the junctions. Figure 7(a) shows a typical temperature dependence of $R_{N}$ for a $4 \mu \mathrm{m}$ wide undoped junction. The resistance decreases with decreasing temperature from $T_{C}$ to about $40 \mathrm{~K}$ as could be expected for a metallic barrier. Below $40 \mathrm{~K}$, the resistance is temperature independent.

The typical temperature dependence of $R_{N}$ of a $\mathrm{La}(5 \%)$ doped junction can be seen in Fig. 7(b). Similar to the case of undoped junctions, the resistance decreases with decreasing temperature for $T>50 \mathrm{~K}$. Below $50 \mathrm{~K}$, there is a striking difference to the undoped junctions, since $R_{N}$ increases with decreasing temperature.

We analyzed the temperature dependence in the lowtemperature regime in more detail. The temperature dependent contribution to the conductivity $\sigma(T)=1 / R_{N}(T)$ $-1 / R_{N}(0 \mathrm{~K})$ against the temperature $T$ in a double logarithmic plot can be seen in Fig. 8. In the temperature range from 0 to $50 \mathrm{~K}$, the data points are on a line with the slope $4 / 3$, resulting in

$$
\sigma(T)\left(\Omega^{-1}\right)=1 / 1.38+8.1 \times 10^{-4} T^{4 / 3} .
$$
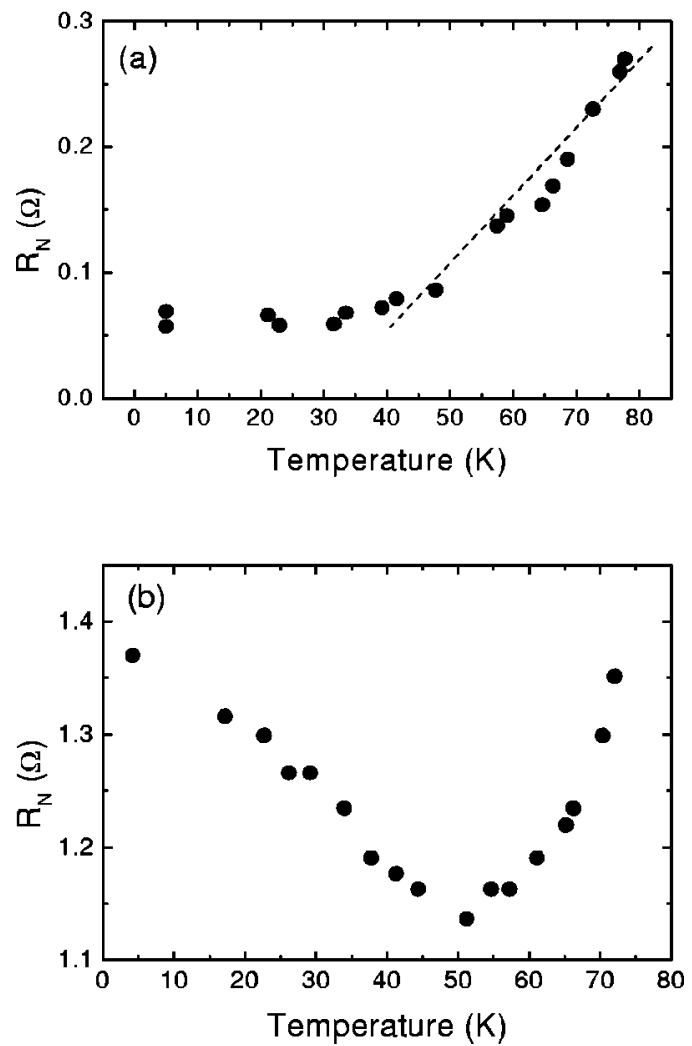

FIG. 7. Temperature dependence of the normal resistance (a) for a $4 \mu \mathrm{m}$ wide undoped junction and (b) for a $3 \mu \mathrm{m}$ wide $\mathrm{La}(5 \%)$ doped junction. The dashed line in (a) sketches the linear dependence of $R_{N}$ between 80 and $40 \mathrm{~K}$.

This behavior can be explained by the GlazmanMatveev theory. ${ }^{18}$ Resonant tunneling via one and two localized states leads to the following dependence of $I$ on $V$ :

$$
\begin{aligned}
& I=\left[\left\langle G_{1}\right\rangle+\left\langle G_{2}(T, 0)\right\rangle+\left\langle G_{2}(0, V)\right\rangle\right] \cdot V, \\
& \left\langle G_{2}(T, 0)\right\rangle=A T^{4 / 3} \quad \text { for } \mathrm{eV} \ll k_{B} T, \\
& \left\langle G_{2}(0, V)\right\rangle=B V^{4 / 3} \quad \text { for } k_{B} T \ll \mathrm{eV} .
\end{aligned}
$$

The temperature and voltage independent part of the conductivity, $G_{1}$, contains the contributions from direct tunneling and tunneling via one localized state. The temperature

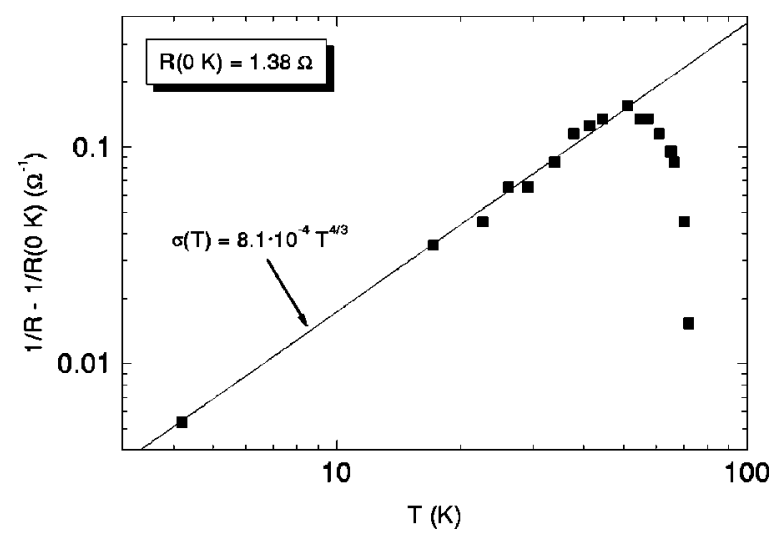

FIG. 8. Temperature dependent contribution to the conductivity of a $3 \mu \mathrm{m}$ wide $\mathrm{La}(5 \%)$ doped junction in a double logarithmic scale. Straight line: Result of the linear regression of the data points from 0 to $50 \mathrm{~K}$. 
and voltage dependent parts, $\left\langle G_{2}(T, 0)\right\rangle$ and $\left\langle G_{2}(0, V)\right\rangle$, respectively, are contributions from resonant tunneling via two localized states. These voltage and temperature dependences have been observed for HTS junctions with different barrier materials, e.g., $\mathrm{PrBa}_{2} \mathrm{Cu}_{3} \mathrm{O}_{7}$ (Refs. 19 and 20) and $\mathrm{SrRuO}_{3}{ }^{21}$

The voltage dependence of the normal resistance due to resonant tunneling via two localized states is expected to become visible for $\mathrm{eV}>k_{B} T$ [see Eq. (3)]. In the case of junctions with $\mathrm{PrBa}_{2} \mathrm{Cu}_{3} \mathrm{O}_{7}$ barriers, the voltage dependence was fitted to the Glazman-Matveev theory between 25 and $60 \mathrm{mV}$ at $4.2 \mathrm{~K}^{20}$ In the case of our IEJs with a typical $R_{N}$ of $1 \Omega$, this corresponds to currents of $25-60 \mathrm{~mA}$, which are in the order of the current densities of the YBCO electrodes. Therefore for high bias currents we observe an increase of the resistivity due to dissipation in the electrodes which dominates a possible decreasing resistivity due to resonant tunneling via two localized states.

Summarizing, the normal resistances of undoped junctions decrease until they saturate at about $40 \mathrm{~K}$. The normal resistances of $\mathrm{La}(5 \%)$ doped junctions as well decrease with decreasing temperature down to $50 \mathrm{~K}$ but start to increase with decreasing temperature below $40 \mathrm{~K}$. The temperature dependence of $R_{N}$ below $40 \mathrm{~K}$ can be explained by resonant tunneling via two localized states.

In order to draw conclusions to the transport of Cooper pairs we analyzed the temperature dependence of the critical current. Figure 9 shows typical $I_{C}(T)$ curves for an undoped [Fig. 9(a)] and a $\mathrm{La}(5 \%)$ doped junction [Fig. 9(b)]. The shapes of the curves are almost linear with an increase of the slope for low temperatures and near $T_{C}$. The characteristics cannot be fitted by an exponential law as expected for a superconductor-normal metal-superconductor (SNS) junction $^{22,23}$ and do not saturate at low temperatures as expected for a tunnel junction. ${ }^{24}$ A so called "quasilinear" temperature dependence of the critical current, similar to our data, was observed for many HTS junctions. ${ }^{25}$ For example, the $I_{C}(T)$ dependences of HTS junctions with Au interlayers were fitted to a superconductor-constriction-superconductor $(\mathrm{ScS})$ model. $^{26}$

For the undoped junctions, the interpretation of the $I_{C}(T)$ data is difficult because the $I-V$ characteristics are flux-flow-like below $60 \mathrm{~K}$ (see Fig. 4). Therefore for the main part of the temperature region below $T_{C}$, the critical current may be correlated with the pinning force of vortices and not with the Josephson-coupling energy. Due to these considerations, it is not reasonable to compare the $I_{C}(T)$ data of the undoped junctions with existing theories for Josephson junctions. Therefore for further analysis we regard only $I_{C}(T)$ curves of $\mathrm{La}(5 \%)$ junctions.

\section{B. Model for the current transport}

The temperature dependences of $I_{C}$ and $R_{N}$ of undoped and $\mathrm{La}(5 \%)$ doped junctions described in the previous section lead us to a consistent picture of the barrier for both types of junctions which is shown in Fig. 10. The barrier consists of a series connection of an insulator with pinholes (constrictions) and a superconductor with reduced $T_{C}\left(S^{\prime}\right)$. In the equivalent scheme in Fig. 10, it can be seen that there
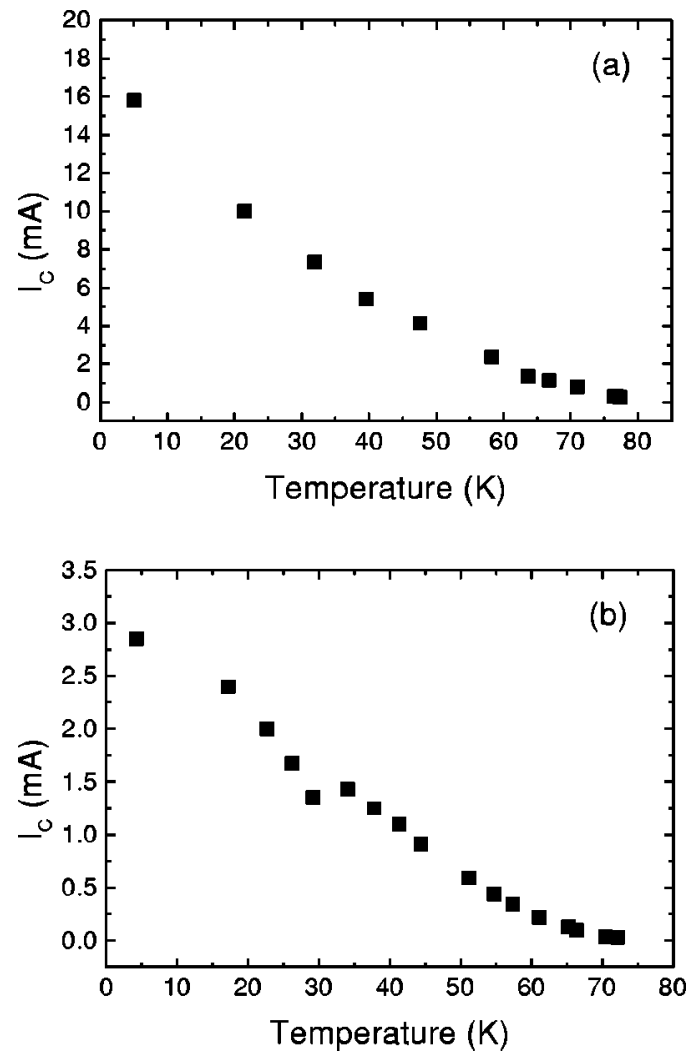

FIG. 9. Temperature dependence of the critical current (a) for a $4 \mu \mathrm{m}$ wide undoped junction and (b) for a $3 \mu \mathrm{m}$ wide $\mathrm{La}(5 \%)$ doped junction.

exist several channels in parallel in the insulating region $\left(R_{0}-R_{2}\right.$ and $\left.R_{\text {const }}\right)$ and one metallic channel $\left(R_{\text {metal }}\right)$ in the $S^{\prime}$ layer. The dominant channel in the insulating region depends on the density of constrictions, their diameter, the electrical properties, and the thickness of the insulator. Direct tunneling as well as resonant tunneling are transport mechanisms for the quasiparticles if the insulator contains localized states. Concerning the supercurrent, it is still an open question whether Cooper-pair transport via localized states is possible in HTS junctions or if pair breaking due to Coulomb (a)

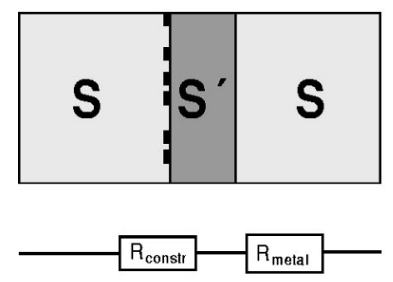

(b)

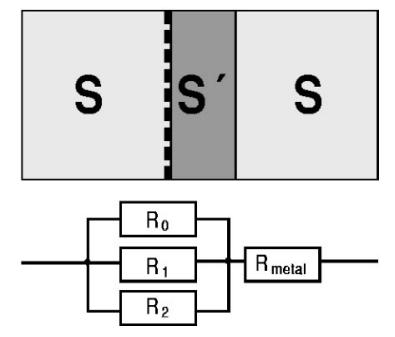

FIG. 10. Scheme of the barrier in the IEJs and equivalent scheme of the quasiparticle conductivity (a) for undoped junctions and (b) La(5\%) doped junctions. The barrier consists of an insulator (black area) with pinholes (constrictions) and an $S^{\prime}$ layer. $R_{0}$ : resistivity due to direct tunneling; $R_{1}$ : resistivity due to resonant tunneling via one localized state; $R_{2}$ : resistivity due to resonant tunneling via two localized states; $R_{\text {const }}$ : boundary resistance in the constriction; and $R_{\text {metal }}$ : resistivity of the $S^{\prime}$ layer above its $T_{C}$. 


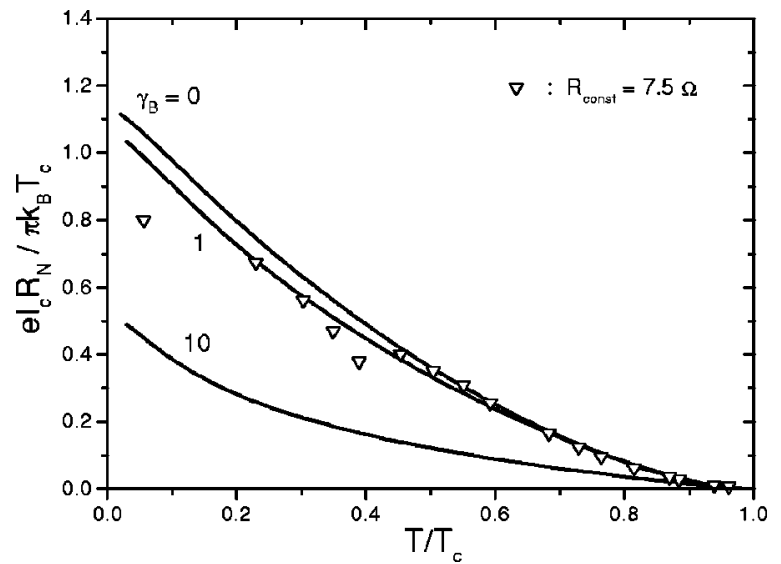

FIG. 11. Comparison of the $I_{C}(T)$ data of a $3 \mu \mathrm{m}$ wide $\mathrm{La}(5 \%)$ doped junction with the ScNS model (Ref. 28) Straight lines: Calculation of the normalized $I_{C} R_{N}$ in the ScNS model for $D=1, d_{N} / \xi=1, \gamma=1$, and $\gamma_{B}$ $=0,1,10 . \nabla$ : Normalized $I_{C}(T) R_{N}$ data with $R_{\text {const }}=5.7 \Omega$ and $T_{C}=75 \mathrm{~K}$.

repulsion makes it impossible. ${ }^{27}$ In our junctions, it is reasonable to assume that the Cooper-pair transport occurs via the constrictions in the insulator.

For the undoped junctions, no contribution from resonant tunneling via two localized states was observed. Therefore it is reasonable to assume that the density and the diameter of the constrictions allow Cooper-pair as well as quasiparticle transport through the constrictions. The resistivity of the constrictions is so low $\left(R_{\text {const }} \ll R_{0,1,2}\right)$ that the current transport through the insulator can be neglected. At high temperatures, the normal resistance is determined by the resistivity of the $S^{\prime}$ layer, $R_{\text {metal }}$. Below $40 \mathrm{~K}, R_{\text {const }}$ exceeds $R_{\text {metal }}$ and $R_{N}$ is determined by the temperature independent interface resistance of the constrictions.

In the case of $\mathrm{La}(5 \%)$ doped junctions, the contribution from resonant tunneling via localized states to the quasiparticle transport can be observed in the temperature dependence of $R_{N}$ (Fig. 8). This means that the diameter and the density of the constrictions are so small that the resistance of the constriction channels always exceeds the resistivity of the resonant-tunneling channels $\left(R_{\text {const }} \gg R_{0,1,2}\right)$ [Fig. 11(b)].

The Cooper-pair transport occurs only via the constrictions. This enables us to compare the $I_{C}(T)$ data to calculations of a simplified model which describes the current transport through a series connection of a normal conducting $N$ layer and a constriction (ScNS model). ${ }^{28}$ Within this model, the NS sandwich is characterized by the suppression parameters $\gamma, \gamma_{B} \cdot{ }^{29}$ These parameters describe the proximity effect in the NS bilayer and are defined as

$$
\gamma_{B}=\frac{R_{B}}{\rho_{N} \xi_{N}}, \quad \gamma=\frac{\rho_{S} \xi_{S}}{\rho_{N} \xi_{N}},
$$

where $R_{B}$ is the specific resistance of the NS interface, $\rho_{N, S}$ are the normal-state resistivities, and $\xi_{N, S}$ are the coherence lengths of the $\mathrm{N}$ and $\mathrm{S}$ materials, respectively. These parameters can be understood as follows: The parameter $\gamma_{B}$ describes the effect of the boundary transparency between these layers. $\gamma$ is a measure of the strength of the proximity effect between the $\mathrm{N}$ and $\mathrm{S}$ metals, i.e., it describes the diffusion of Cooper pairs from $\mathrm{S}$ into $\mathrm{N}$ across the interface. In our junc- tions, a nonzero $\gamma$ also describes that we do not have a sharp NS interface because the ion induced defects will decrease continuously in the superconductor at the interface which leads to a gradually increased order parameter. The constriction is characterized by the transmission coefficient $D$ which accounts for the scattering of Cooper pairs in the constriction.

Figure 11 shows the normalized $I_{C} R_{N}$ product calculated for $\gamma=1$ and different values of $\gamma_{B}$. The transparency $D$ is assumed to be $D=1$ because for $D<1$ the curves saturate at low temperatures what is not observed for our data. The shape is similar to the $I_{C}(T)$ curve of the $\mathrm{La}(5 \%)$ junction in Fig. 9(b). In order to compare our data quantitatively to the model, we would have to take the resistance of the constriction, $R_{\text {const }}$, which we are not able to extract from our experimental $R_{N}(T)$ data due to the existence of parallel conducting channels.

The open triangles in Fig. 11 show the normalized $I_{C} R_{N}$ product of the $\mathrm{La}(5 \%)$ doped junction if we choose $R_{\text {const }}$ $=5.7 \Omega$. The data fit well to the curve corresponding to $\gamma$ $=1$ and $\gamma_{B}=1$. Similar agreement of our data could be obtained by comparing it to calculations with higher values of $\gamma$ and $\gamma_{B}$ which reduce the normalized $I_{C} R_{N}$ product. Because of the ambiguity of estimating $R_{\text {const }}$ and the abovementioned fact that the ScNS model only describes a sharp NS interface, it is not reasonable to make a quantitative fit and draw conclusions from the parameters. However, a qualitative agreement of our data to the ScNS model is obtained.

Furthermore, the ScNS model predicts an excess current in the $I-V$ characteristics due to Andreev reflections at the interfaces. ${ }^{28}$ As mentioned in Sec. III B, due to self-field effects, a dc supercurrent exists which cannot be discriminated from an excess current due to Andreev reflections. Therefore it is not reasonable to discuss the existence of excess currents in terms of the ScNS model.

In summary, the model for our barrier sketched in Fig. 10 qualitatively describes the $R_{N}(T)$ dependence and the $I_{C}(T)$ dependence of the undoped and $\mathrm{La}(5 \%)$ doped junctions. Since we did not observe a material with different lattice constants at the interface in TEM studies, the layers with different electrical properties have to be identified with orthorhombic YBCO with different levels of damage. The fact that the properties of our junctions are stable in time and against $\mathrm{O}_{2}$-annealing treatments suggests cation disorder instead of disorder in the oxygen sublattice to be the main lattice defect. Especially, the constrictions have to be identified with small areas with undisturbed YBCO in a cation disordered YBCO matrix.

It is difficult to distinguish between an $N$ layer and an $S^{\prime}$ layer if an additional interface resistance is present, but it is more likely to attribute the metallic properties to an $S^{\prime}$ layer because YBCO becomes a disordered hopping insulator as soon as $T_{C}$ is completely suppressed by the presence of defects. This would lead to a strong increase of $R_{N}$ at low temperatures.

According to our model, the presence of La reduces the density and the diameter of the constrictions. That means that La homogenizes the engineered interface. This is partly 
in agreement with the TEM investigations of Wen et al. ${ }^{11}$ Although they detected a different barrier layer in their IEJs, they also observed a homogenization of their barrier in the presence of La.

\section{SUMMARY}

IEJs have been fabricated by using $\mathrm{YBa}_{2} \mathrm{Cu}_{3} \mathrm{O}_{7}$ as well as $\mathrm{YBa}_{2-x} \mathrm{La}_{x} \mathrm{Cu}_{3} \mathrm{O}_{7}$ for the superconducting electrodes, and the role of $\mathrm{La}$ in our fabrication process has been investigated. The critical currents of the $\mathrm{La}(5 \%)$ doped junctions are about one order of magnitude lower as the critical currents of the undoped junctions, and the normal resistances are about one order of magnitude higher. We investigated different concentrations of La and found out that the properties of the junctions cannot be continuously adjusted by the La content, but there exists a threshold value of about $5 \%$ for La to influence the junction properties. This shows that La is prerequisite for the modification of the barrier but does not form the barrier by local doping of the interface.

At current densities where the junctions are in the small junction limit, the static $I-V$ characteristics as well as the $I-V$ characteristics under microwave irradiation can be well described with the RCSJ model. The $I_{C}(H)$ patterns in this regime are similar to the ideal Fraunhofer pattern. Junctions with $w / \lambda_{J}$ in the order of 4 exhibit excess currents which can be attributed to self-field effects. In the case of the undoped junctions, the current densities are so high that ideal RCSJ behavior can only be observed near $T_{C}$. The La(5\%) doped junctions are small junctions above about $50 \mathrm{~K}$ and are therefore more suitable for cryoelectronic applications.

The current transport in our junctions can be explained by a barrier consisting of a series connection of an $S^{\prime}$ layer and an insulator, containing microshorts (constrictions) and localized states. We suggest these layers and the constrictions to be YBCO with different degrees of cation disorder induced by the ion-milling treatment. For the undoped junctions, the resistance of the constrictions is so low that the quasiparticle transport as well as the Cooper-pair transport occurs via the constrictions in the insulator. The presence of La reduces the number density and the diameter of the constrictions. As a result, the quasiparticle-current transport in the $\mathrm{La}(5 \%)$ doped junctions occurs by resonant tunneling via localized states in the insulating layer. Since the $I_{C}(T)$ dependence can be fitted to an ScNS model, it is reasonable that the Cooper-pair transport occurs via the constrictions and not by resonant tunneling via localized states across the insulator.

\section{ACKNOWLEDGMENT}

The work was supported in part by the German DFG Project No. Si 704/1-1.

${ }^{1}$ B. H. Moeckly and K. Char, Appl. Phys. Lett. 71, 2526 (1997).

${ }^{2}$ B. D. Hunt, M. G. Forrester, J. Talvacchio, and R. M. Young, IEEE Trans. Appl. Supercond. 9, 3362 (1999).

${ }^{3}$ Y. Soutome, T. Fukazawa, A. Tsukamoto, Y. Tarutani, and K. Takagi, 1999 International Workshop on Superconductivity, Hawaii, Extended Abstracts (1999), p. 113.

${ }^{4}$ A. Fujimaki, K. Kawai, N. Hayashi, M. Horibe, M. Maruyama, and H. Hayakawa, IEEE Trans. Appl. Supercond. 9, 3436 (1999).

${ }^{5}$ T. Satoh, M. Hidaka, and S. Tahara, IEEE Trans. Appl. Supercond. 9, 3141 (1999)

${ }^{6}$ R. Dittmann, J.-K. Heinsohn, A. I. Braginski, and C. L. Jia, IEEE Trans. Appl. Supercond. 9, 3440 (1999).

${ }^{7}$ T. Satoh, J. G. Wen, M. Hidaka, S. Tahara, N. Koshizuka, and S. Tanaka, International Workshop on Superconductivity, Hawaii, Extended Abstracts (1999), p. 199.

${ }^{8}$ W. C. Steward, Appl. Phys. Lett. 12, 277 (1968); D. E. McCumber, J. Appl. Phys. 39, 3113 (1968).

${ }^{9}$ Y. Huang, K. L. Merkle, B. H. Moeckly, and K. Char, Physica C 314, 36 (1999).

${ }^{10}$ C. L. Jia, M. I. Faley, U. Poppe, and K. Urban, Appl. Phys. Lett. 67, 3635 (1995).

${ }^{11}$ J. G. Wen, N. Koshizuka, S. Tanaka, T. Satoh, M. Hidaka, and S. Tahara, Appl. Phys. Lett. 75, 2470 (1999).

${ }^{12}$ J.-K. Heinsohn, R. H. Hadfield, and R. Dittmann, Physica C 326-327, 157 (1999).

${ }^{13}$ C. Horstmann, P. Leinenbach, A. Engelhardt, R. Gerber, C. L. Jia, R. Dittmann, U. Memmert, U. Hartmann, and A. I. Braginski, Physica C 302, 176 (1998).

${ }^{14}$ A. Marx, K.-H. Husemann, B. Meyer, T. Nissel, R. Gross, M. A. J. Verhoeven, and G. J. Gerritsma, Appl. Phys. Lett. 64, 241 (1994).

${ }^{15}$ A. P. B. Sinha, I. S. Mulla, and M. R. Chandrachood, in Chemical and Structural Aspects of High Temperature Superconductors (World Scientific, Singapore, 1988).

${ }^{16}$ A. Barone and G. Paternó, Physics and Applications of the Josephson Effect (Wiley, New York, 1982).

${ }^{17}$ J. R. Waldram, A. B. Pippard, and J. Clarke, Philos. Trans. R. Soc. London, Ser. A 268, 265 (1970).

${ }^{18}$ L. I. Glazman and K. A. Matveev, Sov. Phys. JETP 67, 1276 (1988).

${ }^{19}$ M. A. J. Verhoeven, G. J. Gerritsma, H. Rogalla, and A. A. Golubov, Appl. Phys. Lett. 69, 848 (1996).

${ }^{20}$ A. Engelhardt, R. Dittmann, and A. I. Braginski, Phys. Rev. B 59, 3815 (1999).

${ }^{21}$ R. Dömel, C. Horstmann, M. Siegel, A. I. Braginski, and M. Yu. Kupriyanov, Appl. Phys. Lett. 67, 1775 (1995).

${ }^{22}$ P. G. de Gennes, Rev. Mod. Phys. 36, 225 (1964).

${ }^{23}$ M. Yu. Kupryanov, Sov. J. Low Temp. Phys. 7, 342 (1981)

${ }^{24}$ V. Ambegaokar and A. Baratoff, Phys. Rev. Lett. 10, 486 (1963).

${ }^{25}$ K. A. Delin and A. W. Kleinsasser, Supercond. Sci. Technol. 9, 227 (1996).

${ }^{26}$ M. Bode, M. Grove, M. Siegel, and A. I. Braginski, J. Appl. Phys. 80, 6378 (1996).

${ }^{27}$ L. I. Glazman and K. A. Matveev, JETP Lett. 49, 659 (1989).

${ }^{28}$ A. A. Golubov, V. M. Krasnov, and M. Yu. Kupryanov, J. Low Temp. Phys. 106, 249 (1997).

${ }^{29}$ M. Yu. Kupryanov and V. F. Lukichev, Sov. Phys. JETP 67, 1163 (1988). 